\title{
Identification of ecdysone response elements by analysis of the Drosophila Eip28/29 gene
}

\author{
Lucy Cherbas, ${ }^{1}$ Keesook Lee, ${ }^{1}$ and Peter Cherbas \\ Program in Molecular, Cellular, and Developmental Biology, Institute for Molecular and Cellular Biology, and the \\ Department of Biology, Indiana University, Bloomington, Indiana 47405 USA
}

\begin{abstract}
We have identified ecdysone-response elements (EcREs) by studying regulation of the steroid-responsive Drosophila Eip28/29 gene. First, functional assays of deletion mutants identified large sequence regions required for the response; then a blotting method using the specifically labeled steroid receptor as probe identified receptor-binding regions. Three short receptor-binding regions near Eip28/29 have been identified: Prox and Dist [521 and 2295 nucleotides, respectively, downstream of the poly(A) site] are probably required for the Eip28/29 response in cell lines; Upstream $(-440)$ is unnecessary for that response. We have also demonstrated that an EcRE-containing region from hsp27 contains a receptor-binding site. Each of these four receptor-binding regions functions as an EcRE when placed upstream of an ecdysone nonresponsive promoter and each contains an imperfect palindrome, suggesting the consensus $5^{\prime}-\mathrm{RG}$ (GT)TCANTGA(CA)CY-3'. Furthermore, a synthetic 15-bp fragment containing an imperfect palindrome similar to the consensus is a fully functional EcRE. The presence of any of the EcREs leads, in the absence of hormone, to depressed gene expression. When hormone is added, it relieves this repression and causes additional activation. The similarity of the EcRE sequence to response elements for estrogen, thyroid hormone, and retinoic acid receptors suggests that the steroid receptors and their signal transduction mechanisms have been strongly and broadly conserved.
\end{abstract}

[Key Words: Ecdysone response elements; ecdysone receptor; steroid hormones; Drosophila melanogaster; Eip28/29; Kc cells]

Received August 27, 1990; revised version accepted October 26, 1990.

Ecdysone is the steroid molting hormone of arthropods; it regulates the molting cycle and provokes the remarkable developmental events associated with metamorphosis. Ever since the pioneering work of Clever (1964) and Ashburner (see, e.g., Ashburner et al. 1974) on hormone-induced puffing in salivary gland chromosomes suggested that ecdysone might act by regulating transcription, it has been attractive to suppose that all of the steroid hormones are functionally related and that they control transcription by similar mechanisms. If so, studies of ecdysone action in Drosophila should contribute significantly to our understanding of the mechanistic bases of the developmental specificity of steroid effects.

Strong evidence has accumulated demonstrating that the vertebrate steroids do share a common mechanism of action, a mechanism that depends on a conserved family of ligand-dependent transcription factors, the steroid receptors. Each receptor binds its cognate ligand tightly and specifically and, in the presence of steroid, these zinc-finger proteins also bind to specific hormone response elements (HREs) in the vicinity of responsive genes. The HREs function as hormone-dependent tran-

${ }^{1}$ The two first authors are listed alphabetically. scriptional enhancers. The HREs for the vertebrate steroid hormones constitute a family of short, more-or-less palindromic, and closely related DNA sequences, which are strongly conserved among vertebrates (for review, see Evans 1988; Beato 1989; Carson-Jurica et al. 1990).

In the case of ecdysone, considerable indirect evidence is consistent with a similar mechanism of action, but direct evidence for transcriptional regulation by ecdysone receptors has been elusive. Thus, active ecdysones are known to bind with appropriate affinities to a nuclear protein (or proteins) present at low abundance in responsive cells and thought to be receptor(s) (Maroy et al. 1978; Yund et al. 1978; Sage et al. 1982; Cherbas et al. 1988), and number of genes with the response characteristics of immediate hormone targets ("early effects") are known (for review, see Cherbas et al. 1986a; Ashburner 1990; Segraves and Richards 1990). Using one of these genes (hsp27), Riddihough and Pelham (1987) described an upstream sequence sufficient to confer hormonal inducibility in transient assays. They proposed, but did not establish, that a protein binding that sequence is the ecdysone receptor.

Our goal has been to identify ecdysone-response elements (EcREs); to do so we have begun by identifying sequences necessary for the hormonal regulation of the gene Eip28/29, which is ecdysone-inducible in some 
Drosophila cell lines (Savakis et al. 1980, 1984) and in some larval tissues (Andres 1990). In cell lines this induction is known to be transcriptional and rapid /Savakis et al. 1980, 1984; Bieber 1986); in these respects, its regulation can be considered analogous to that of the "early puffs" in the salivary gland. The region just upstream of Eip28/29 is transcriptionally complex; the genes $z 600, g d l$, and Eip28/29 are represented by overlapping transcription units on a single DNA strand, but the three genes are expressed at very different times and places in development (Schulz et al. 1989; Schulz and Butler 1989; Schulz and Miksch 1989|. Of particular interest in the present context is the fact that $g d l$, whose $3^{\prime}$-noncoding region overlaps the $5^{\prime}$ end of Eip28/29, is expressed at a low level in Kc cells but is entirely unaffected by ecdysone (Schulz et al. 1989). Hence, any satisfactory explanation for the ecdysone responsiveness of Eip28/29 must also account for the fact that its overlapping upstream neighbor is hormone refractory.

In the present communication we describe the sequences of several functional EcREs. These elements have been identified by their roles in the cis regulation of Eip28/29, by their ability to bind ecdysone receptors, and by their ability to confer hormonal inducibility on suitable reporters. Taken together, these sequences (along with the sequence described by Riddihough and Pelham 1987) provide a consistent picture of the nature of an EcRE. Both the fact that the ecdysone receptor binds to EcREs and the sequence content of the EcREs themselves provide strong support for the idea that ecdysone and its receptor are elements of a highly conserved signal response system very similar to that of the vertebrate steroid hormones.

\section{Results}

We began by using deletion mutagenesis and transient expression in Drosophila cells to map sequences involved in the ecdysone responsiveness of Eip28/29. Both the $\mathrm{Kc167/M} 3$ and EH34A3/M3 cell lines show excellent Eip28/29 induction, and both yield good gene expression following transfection; we have used both lines, with indistinguishable results, in the experiments that follow (see Materials and methods).

All of the cell lines we have tried share the serious handicap that simple exposure to the conditions of transfection reduces the magnitude of the ecdysone response as judged by Eip28/29 induction. Thus, after any transfection (see, e.g., Fig. 1) or mock transfection (no exogenous DNA; data not shown) the endogenous Eip28/29 gene is induced only 3- to 4-fold (Fig. 1, lanes 3-6) rather than 10- to 15 -fold as it is under normal conditions (lanes 1-2). Although this constriction of the dynamic range of the response may eventually prove limiting, it has not, in the present instance, prevented us from identifying regions with strong effects on the hormone response.

For our initial experiments (Fig. 2), plasmids were constructed in which Eip28/29-flanking sequences of varying extents were combined with either of two re-

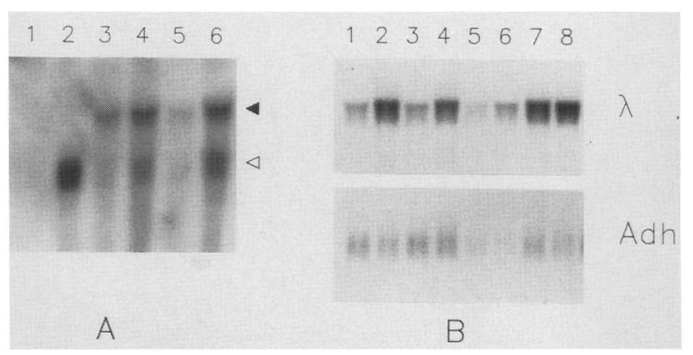

Figure 1. Induction of tagged Eip28/29 genes by ecdysone. EH34A3/M3 cells were transfected with plasmids bearing tagged Eip28/29 genes, and RNA was isolated $24 \mathrm{hr}$ later for analysis by Northern blots; during the final $4 \mathrm{hr}$ of incubation, cells for some lanes were treated with hormone $\left(10^{-6} \mathrm{M} 20\right.$-hydroxyecdysone). (For the structures of the plasmids, see Fig. 2.) (A) The blot was probed with a single-stranded Eip28/29-specific DNA probe to reveal transcripts from both the exogenous (solid arrowhead) and the endogenous (open arrowhead) genes. (Lanes 1 and 2) Untransformed cells; (lanes 3 and 4) construct 12; (lanes 5 and 6) construct 1. (Lanes 1, 3, and 5) No hormone treatment; (lanes 2, 4, and 6) hormone-treated. $\langle B|$ Cells were cotransformed with an equimolar mixture of the indicated plasmid and Eip188-Adh as internal control /see Materials and methods). Duplicate blots were probed with a single-stranded RNA probe for $\lambda(t o p)$ and a single-stranded RNA probe for $A d h$ (bottom). (Lanes 1 and 2) Construct 1; (lanes 3 and 4) construct 4; (lanes 5 and 6) construct 10; (lanes 7 and 8) construct 12 . (Lanes 1, 3, 5, and 7) no hormone treatment; (lanes 2, 4, 6, and 8) hormone treated.

porters: (1) a modified Eip28/29 transcription unit tagged, in the $3^{\prime}$-untranslated region, by insertion of a 499-bp fragment of bacteriophage $\lambda$ and detectable by Northern analysis, or (2) the chloramphenicol acetyltransferase (cat) gene.

Table 1 indicates that basal expression (i.e., hormoneindependent expression) does not require an extended 5 -flanking region; constructs containing only $188 \mathrm{bp}$ of 5 '-flanking DNA yield essentially full basal expression. Relative basal expression levels were determined for most of the Eip transcription unit-based constructs shown in Figure 2 by cotransfecting with an Adh-expressing plasmid, normalizing, and comparing the results with construct 1 (see Materials and methods). For construct 1 itself, our impression, on the basis of results from numerous permanently transformed clonal cell lines, is that expression per gene copy is comparable to that for the endogenous gene (data not shown). Save for a very minor decrease in expression when the $5^{\prime}$-flanking region extends upstream of -657 - a decrease that may be related to the presence (and function; data not shown) of the gdl promoter-exceptional levels of basal expression were exhibited by only two constructs, 3 and 12 . Construct 3 lacks introns, and we suspect that its diminished expression reflects instability of the primary transcript. Construct 12 is more interesting. This construct, which has the shortest 3 '-flanking region tested, exhibits reproducibly elevated basal expression (e.g., Fig. 1A, lane 3; Fig. 1B, lane 7), a phenomenon to which we shall return later. 
Figure 2. Deletion analysis of ecdysone inducibility at Eip28/29. (Left) The overlapping transcription units for Eip28/29 (Cherbas et al. 1986b), gdl, and $z 600$ (Schulz et al. 1989; Schulz and Butler 1989) are shown at the top; the axis (in $\mathrm{kb}$ ) represents the entire sequenced region, with the origin taken as +1 of the Eip28/29 transcription unit. [For clarity, we have included only two (short form gdl ${ }^{M}$ and long form $g d l^{F}$ ) of the four $g d l$ transcription units.] Rectangles represent exons; solid rectangles represent coding sequence. The flanking sequences (of Eip28/ 29) are represented by thin open bars. Within the constructs, the site of the 499-bp bacteriophage $\lambda$ insertion in the $3^{\prime}$-noncoding region is indicated. Open rectangular boxes labeled cat represent a 1.6$\mathrm{kb}$ fragment containing a bacterial cat gene; for simplicity it is drawn as if it were the same length as the Eip28/29 transcription unit. In each case the cat fragment is inserted between bases +11 and +2205 of Eip28/29 (where the transcribed region is +1 to +2147 ). (For details of the constructions, see Materials and methods.) Individual construct numbers are given at left; the constructs have been grouped to facilitate comparison. Regions $A-D$, described in the text, are indicated at bottom. (Right) The histogram shows the induction ratio for each construct ( $\lambda$-tagged constructs tested in EH34A3 cells; cat constructs tested in Kc167 cells). Error bars indicate standard errors; the number of independent experiments is shown in brackets. The dashed base line has been drawn at an induction ratio of 1 to emphasize the decreased expression in the null constructs 15 and 16.

Because the cat data are not directly comparable, we cannot eliminate the possibility that sequences internal to the Eip transcription unit contribute to basal expression. We know from experiments using cat reporters that sequences just $3^{\prime}$ of the transcription unit, in region $\mathrm{B}$, are important for full basal expression in Kcl67 and EH34A3 cells (data not shown).

Numerous elements, distributed widely within both flanking sequences, contribute to a full ecdysone response

Figure 1A (lanes 5 and 6) demonstrates that the tagged Eip28/29 gene in construct 1 is fully inducible in the

Table 1. Relative basal expression

\begin{tabular}{lc}
\hline Constructs & Relative basal expression \\
\hline $4,10,11$ & $0.99 \pm 0.08(9)$ \\
$5,6,7$ & $1.26 \pm 0.08(8)$ \\
3 & $0.43 \pm 0.15(3)$ \\
12 & $2.87 \pm 0.32(3)$
\end{tabular}

Basal expression was measured by Northern analysis, and the measurements were corrected by reference to the cotransfected control plasmid Eip188-Adh. Construct 1 was included in each experiment, and all values were normalized by setting the expression of construct 1 to 1.00 (for construct structures, see Fig. 2). Data are presented as relative expression \pm SE (independent experiments). Inter se differences among the four groups were highly significant $(\mathrm{P}<5 \%$ for the first two groups; $\mathrm{P}<<1 \%$ for the others by t-tests). sense that its induction ratio (3.2) is comparable to that for the endogenous gene.

Comparison of the first group of constructs in Figure 2 shows that $3^{\prime}$-flanking sequences are important to the full ecdysone response. Constructs 1 and 4 do not differ significantly; hence, we doubt that significant elements reside to the right of +5882 on the map. However, constructs 10 and 11 (deleted for region $D$ ) show a modestly reduced hormone response. Further $3^{\prime}$ truncation as in construct 12 (deleted for both regions $C$ and $D$ ) leads to a marked additional reduction of the response.

These results show the essentially full induction of constructs 1 and 4 (Fig. 1A, lanes 5 and 6; Fig. 1B, lanes $1-4 \mid$, the modestly diminished induction of construct 10 (Fig. 1B, lanes 5 and 6), and the substantially reduced induction of construct 12 (Fig. 1A, lanes 3 and 4; Fig. 1B, lanes 7 and 8). The weak induction of construct 12 appears to be due, at least in part, to the significant elevation in its basal expression noted earlier.

The second group of constructs was derived from minigenes and lacks introns. Comparison of the ecdysone responses of appropriate pairs (constructs 3 with 1 and 14 with 12) suggests that the introns do not contribute significantly to the response.

A third group of constructs lack the $5^{\prime}$ region $A$. This set (constructs $5-7$ ) can be compared fruitfully with construct 4 , which has the same $3^{\prime}$ extension. The comparison shows that deletion of region $A$ reduces the ecdysone effect modestly.

Finally, to eliminate effects of all the Eip28/29 transcription unit sequences, we have constructed a series of 
cat fusions (constructs $8,9,15$, and 16). Constructs (15 and 16) that contain 188 or 657 bp of $5^{\prime}$-flanking sequence are not induced by ecdysone. Ecdysone treatment leads to a reduction $(\sim 20 \%)$ in their expression. Previously, we had observed just such a reduction with a variety of reporter plasmids assumed to be wholly nonresponsive to ecdysone, for example, pSV2.cat (Gorman et al. 1982; L. Cherbas and N. Rosenthal, unpubl.). We suppose that this is due to some unknown effect of ecdysone on the uptake, retention, or expression of exogenous DNA, and we take an induction ratio of 0.8 to represent a truly "null" response. In contrast, cat constructs ( 8 and 9) that contain the 3 ' regions $B, C$, and $D$ coupled with the same $5^{\prime}$ sequences give ecdysone responses comparable to those of constructs $5-7$, which contain essentially identical flanking regions.

Our results indicate that some element (or elements) within each of the four regions can contribute, at least in certain circumstances, to expression of a full ecdysone response. This is more clearly shown in Table 2 , where the data have been aggregated according to the flanking regions included. Plainly, we observe four distinct levels of inducibility, and these reflect the complement of important flanking regions included. The residual inducibility of group III must be due to elements located within either region $A$ or $B$. We are confident that $B$ contains a weak EcRE because cat constructs containing region $B$ coupled with only 188 bp of upstream sequence have inducibilites comparable to those of constructs 12 and 14 and because constructs containing extensive upstream sequences, but lacking downstream sequences, are not inducible (data not shown).

Evidently, sequences contributing to the ecdysone response, while absent from the transcription unit per se, are widely distributed in the regions flanking Eip28/29, with at least one significant element in the upstream region between -2007 and -658 and three dispersed elements within the 3735 bp downstream of the polyadenylation site. Each of these elements is a candidate to be an EcRE. Given the extent of the DNA to be tested, we elected to proceed by searching for sites that bind the ecdysone receptor.

\section{A blotting assay identifies three receptor-binding sites near Eip28/29}

We devised an approach to facilitate the screening of extended regions of DNA for receptor-binding sites. Briefly, separated restriction fragments were blotted, as native double-stranded molecules, to a cationic membrane. After membrane blocking, receptor-binding sites were probed by incubating the membrane with a crude extract in which the ecdysone receptor had been specifically labeled with the hormone analog 26-[125I]iodoponasterone A (for details, see Materials and methods; Cherbas et al. 1988).

The sensitivity of such a screen ought to be dependent on restriction fragment length. Typically, steroid receptors display only modest DNA-binding specificity, affinities for specific sites being only $\sim 10^{3}$ those for
Table 2. Flanking sequence content defines four induction ratio levels

\begin{tabular}{lllc}
\hline Group & Constructs & $\begin{array}{l}\text { Regions } \\
\text { included }\end{array}$ & $\begin{array}{c}\text { Induction } \\
\text { ratio }\end{array}$ \\
\hline I & $1,3,4$ & A, B, C, D & $3.03 \pm 0.18(23)$ \\
II & 10,11 & A, B, C & $2.40 \pm 0.14(13)$ \\
& $5-9$ & B, C, D & $2.43 \pm 0.18(8)$ \\
III & 12,14 & A, B & $1.38 \pm 0.08(25)$ \\
IV & 15,16 & none & $0.82 \pm 0.04(30)$ \\
\hline
\end{tabular}

Data are presented as induction ratio \pm SE (independent experiments). Constructs and regions are defined in Fig. 2. Inter se differences among the four groups were highly significant ( $P$ $<2 \%$ for every comparison by $\mathrm{t}$-tests).

random sites (see, e.g., Pfahl 1982). If the ecdysone receptor behaves similarly, and if a signal/background of 2 is selected as a practical threshold, fragments should not exceed $1 \mathrm{~kb}$.

Figure 3 shows some typical results. A low-resolution experiment in which the starting DNA $(-657$ to +7100$)$ was cut into mostly large restriction fragments is shown in Figure 3A. Most fragments show nonspecific binding giving an autoradiographic signal roughly proportional to fragment length (a conclusion confirmed by densitometry; data not shown). However, in this instance, it is clear that the two smallest fragments (PstI-d, 648 bp and BglII-d, $432 \mathrm{bp}$ / yield disproportionately strong signals indicative of high-affinity sites. As expected, neither site could have been discerned by inspection of the corresponding large fragments (BglII-a and PstI-a, both $>5 \mathrm{~kb}$. In such low-resolution experiments, only restriction fragments less than $\sim 1 \mathrm{~kb}$ in length are fully informative, although impressions based on somewhat larger fragments have generally survived further testing.

When the restriction fragments are smaller, as in Figure $3 \mathrm{~B}$, the identification of high-affinity sites becomes unambiguous. An experiment involving the 432bp fragment BglII-d from Figure $3 \mathrm{~A}$ is shown in Figure 3B. A series of four bifurcations of the fragment delineate a $27-$ bp region that is evidently critical for binding. This site is designated Prox since it is the more proximal of two downstream binding sites.

All of the binding that we observe, both nonspecific and specific, is due to ecdysone receptor: (1) All of the binding can be blocked by competition with unlabeled steroid (muristerone A, $10^{-4} \mathrm{M}$ ); and (2) previously, it has been demonstrated for similar extracts labeled under comparable conditions that all competition-sensitive binding is due to a protein (or proteins) with the steroid specificity of ecdysone receptor (Cherbas et al. 1988). Thus, unbound (or nonspecifically bound) ligand does not contribute to the signals that we observe; similarly, prior removal of unbound label (by gel filtration) does not affect the results (data not shown).

Using this blotting assay we have looked for receptorbinding sites throughout most of a $9-\mathrm{kb}$ region around Eip28/29, covering virtually all of the sequence from -1929 to +7100 with overlapping restriction fragments of $<1 \mathrm{~kb}$, as summarized in Figure 4. As shown in Figure 
Figure 3. Mapping ecdysone receptorbinding sites by blotting. $(A)$ Coarse mapping of binding sites in the Eip28/29 region. A plasmid whose insert is similar to construct 5 but extends downstream to +7100 (see Fig. 2) was digested with PstI or $B g$ III, and the resulting fragments were separated by electrophoresis $11 \%$ agarose in TAE, $4 \mu \mathrm{g}$ DNA/lane) in duplicate panels. After electrophoresis, one panel was stained with ethidium bromide, and the entire gel was electroblotted. The stained portion of the blot was photographed (left); the unstained portion was incubated with a ${ }^{125}$ I-labeled receptor-ligand mixture (see Materials and methods) and autoradiographed (right). In the map of the insert, fragments are labeled simply $a-d$ in decreasing order of size. There are no sites for

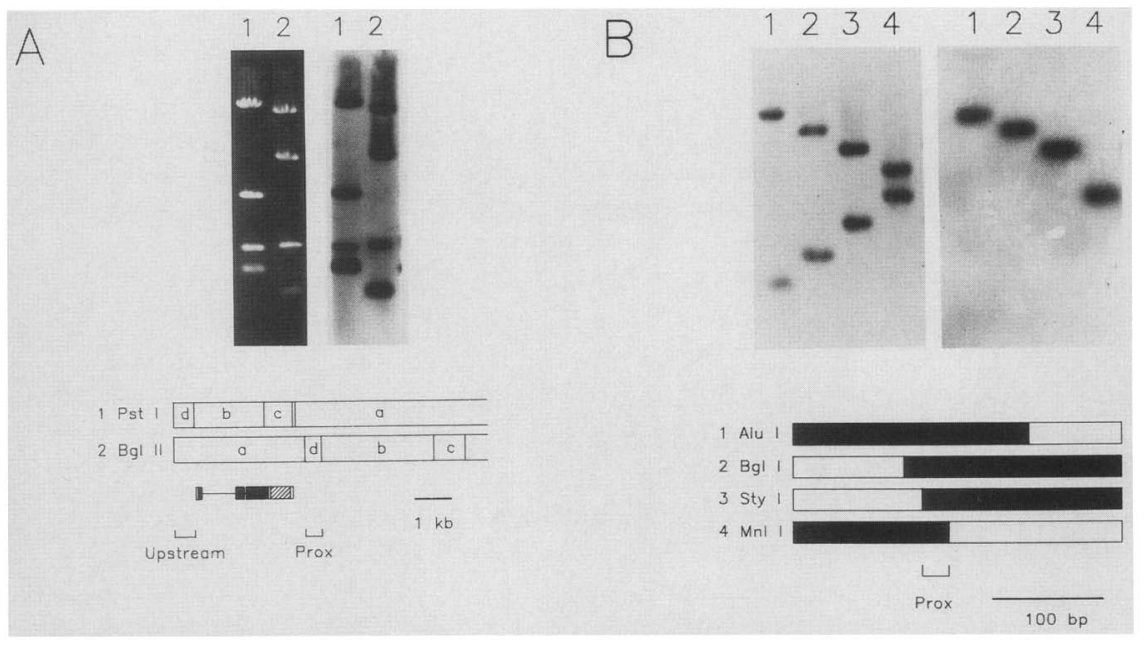
either enzyme in $2.7 \mathrm{~kb}$ of pUC8 vector sequence. The positions of the Eip28/29 transcription unit, the $\lambda$ insert (hatched), and fragments that bound the labeled receptor disproportionately (Upstream and Prox) are indicated below the map. $(B)$ Fine mapping of the binding site Prox. The starting fragment was an isolated 432-bp BglII fragment (fragment $\mathrm{d}$ in $A$ ). To control for blotting efficiency and for the effects of washing, all operations were carried out in parallel on two DNA samples: unlabeled DNA and "tracer DNA" that had been labeled with ${ }^{32} \mathrm{P}$ by nick translation. Aliquots of each DNA $(0.2 \mu \mathrm{g})$ were digested with the enzymes indicated and electrophoresed $\left(8 \%\right.$ polyacrylamide in TBE); the entire gel was electroblotted. The unlabeled panel was incubated with ${ }^{125}$ I-labeled receptor-ligand complex, and washed; the tracer panel was autoradiographed, incubated, and washed similarly, except that cell extract and ${ }^{125}$ I-labeled steroid were omitted from the incubation. Both panels were visualized by autoradiography. (Left) A 3-hr exposure (with intensifying screen) of the tracer panel after incubation and washing showing the two bands that result from cleavage with each restriction enzyme. [An autoradiogram of the same panel prior to incubation and washing appeared identical (not shown).] (Right) A 48-hr exposure (with intensifying screen) of the ${ }^{125}$ I-labeled portion; it demonstrates that only one of each pair of restriction fragments contains a receptor-binding site. The overlap of the binding-positive regions $(27 \mathrm{bp}$ ) defines Prox.

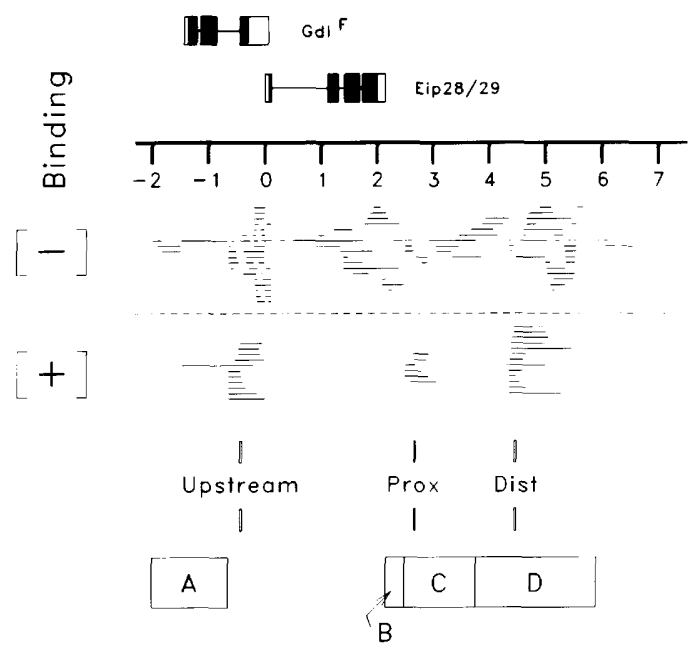

Figure 4. A survey of the Eip28/29 region for ecdysone receptor-binding sites. Each thin horizontal line shows the extent of a restriction fragment that has been tested, by experiments similar to those in Fig. 3, for ecdysone receptor binding. Fragments above the dashed line exhibited no high-affinity binding; those below it exhibited clear evidence of one or more high-affinity sites. Alignments of these fragments identified the three binding sites Upstream, Prox, and Dist, whose positions and extents are indicated below. For orientation, the transcription units for Eip28/29 and $g d l^{F}$ and the positions of regions $A-D$ are given as in Fig. 2.
4, three sites within this large region tested positive: Upstream $(-457$ to -415$)$; Prox $(+2651$ to +2681 , within region $C)$; and Dist $(+4433$ to +4469 , within region $D$ ). The sequences of these sites are shown in Figure 5 .

For comparison, we applied the same assay to the upstream region of $h s p 27$ in which a 23 -bp region $\mid-551$ to -529; Riddihough and Pelham 1987/ confers ecdysone responsiveness. Our survey covered the $5^{\prime}$-flanking region of $h s p 27$ from -757 to -124 and revealed high-affinity binding due to a site (or sites) within the region -562 to -514 , a region that includes the element as described previously (data not shown).

Sequences of the four binding fragments (including the 23-bp hsp27 element as described by Riddihough and Pelham 1987) are presented in Figure 5A, where they have been aligned to emphasize an obvious common element: Each fragment contains at least one copy of the sequence 5 '-TGA $|\mathrm{CA}| \mathrm{C}-3^{\prime}$, usually followed by two pyrimidines, as the right arm of an imperfect palindrome with a single nucleotide at the center of symmetry. When a fragment containing Upstream was cut with MseI (recognition site T/TAA), to disrupt the left half of the palindromic region, binding was eliminated (data not shown), supporting the idea that sequences near the conserved box are significant for binding. Further evidence in support of that conclusion is discussed below. Dist contains two copies of the conserved palindrome, 


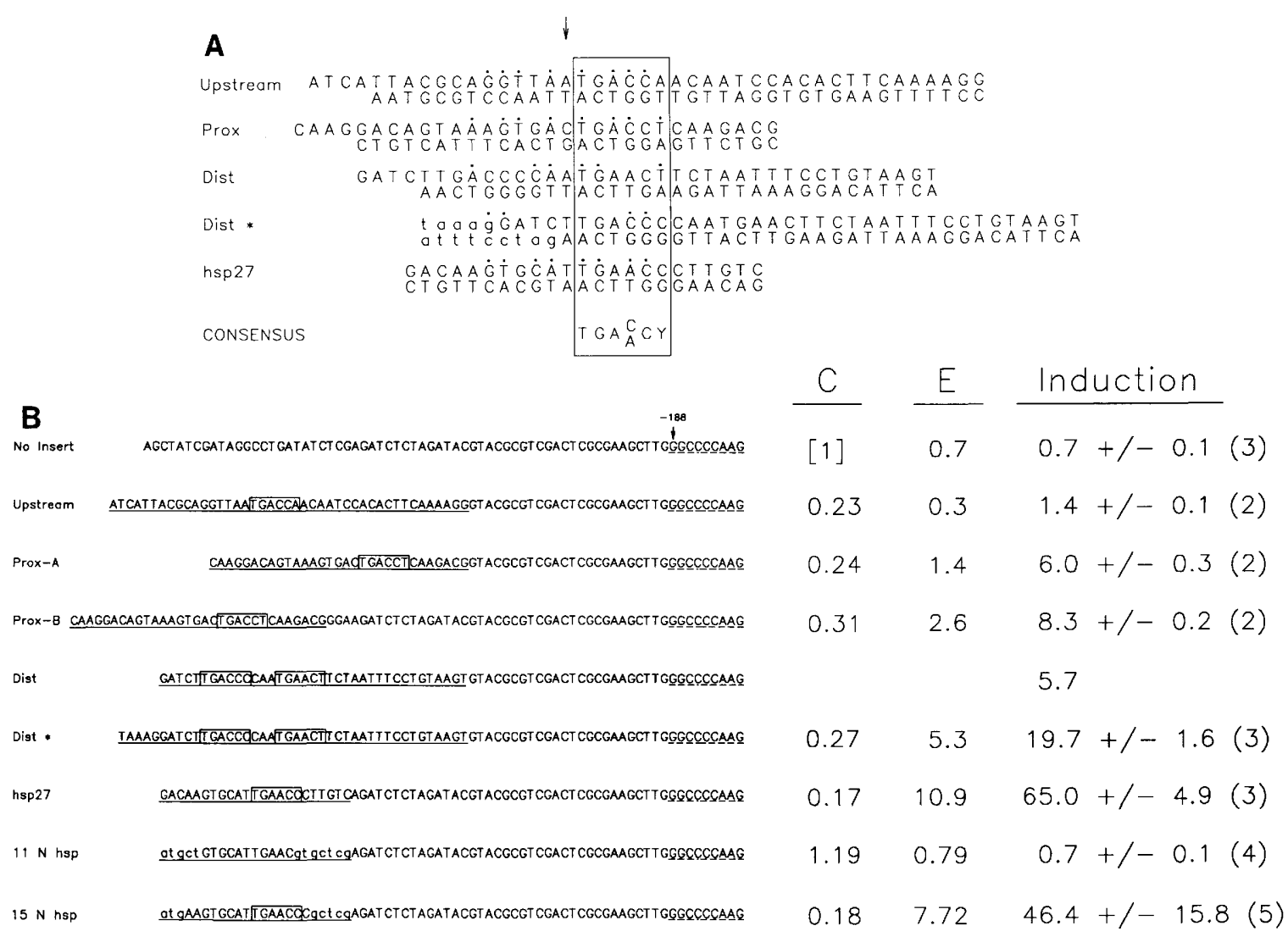

Figure 5. Sequences and functional tests of fragments containing EcREs. (A) Sequences are shown for the three ecdysone receptorbinding sites Upstream, Prox, and Dist, defined by the experiments depicted in Figs. 3 and 4 . Both strands are shown, to indicate the structures of their termini. Dist ${ }^{\star}$ is an alternate alignment of Dist, drawing attention to the presence of a more proximal TGACC; adjacent sequence (not part of the smallest binding-positive fragments) has been given in lowercase letters to indicate that this element is also partially symmetric. The $h s p 27$ region also contains a receptor-binding site (see text). Its sequence corresponds (left to right) to bases -529 to -551 of $h s p 27$ (Riddihough and Pelham 1986). The vertical arrow (top) indicates the center of symmetry of each partial dyad; dots are shown above bases that contribute to this symmetry. The box encloses a hexameric half-palindrome, for which the consensus sequence is indicated below. $(B)$ Each of the sequences shown in $A$ was inserted into X-188-cc-cat (see text) and tested for activity as an EcRE. (Left) The sequence of the inserted fragment (solid underline), of the intervening polylinker, and of a portion of the Eip28/29 promoter fragment (dashed underline); base - 188, the $5^{\prime}$ end of the Eip28/29 fragment, is indicated by an arrow (top). Each copy of the consensus half-palindrome is boxed (as is the functional cognate in Upstream). Prox was inserted into two different sites with results shown as Prox-A and Prox-B. Dist was inserted both as a shorter fragment (Dist) and as the 5-bp longer fragment Dist ${ }^{*}$. The constructs $11 \mathrm{~N} h s p$ and $15 \mathrm{~N} h s p$ contain synthetic oligonucleotides designed to differ (lowercase letters) from the hsp27 23-mer at their ends. Thus, their central bases (uppercase) are identical to those of the $h s p 27$ sequence and either contain (15 $\mathrm{N} h s p$ ) or do not contain (11 N hsp) a full consensus palindrome. Tabular data (right) summarize expression from each construct. The data are derived from CAT activities following transient expression in Kc167/M3 cells. (C) No hormone treatment; (E) 24-hr treatment with $10^{-6}$ M 20-hydroxyecdysone. Induction means induction ratio (E/C). The unmodified vector was included in each experiment, and expression levels were normalized to its expression in untreated cells. Mean values, standard deviations, and the number of experiments (in parentheses) are shown.

as indicated by the two alignments drawn in Figure 5A. Appropriate digestions can release either Dist ${ }^{*}$, which contains two "complete" palindromes or Dist $15 \mathrm{bp}$ shorter), in which the left arm of the more proximal dyad is truncated.

To establish that the fragments shown in Figure 5A contain all of the sequence necessary to give high-affinity binding, we subcloned them into pUC18 or pUCLl (see Materials and methods) and tested the resulting plasmids for receptor binding. The tested fragments were precisely those shown in Figure 5A (with any $5^{\prime}$ extensions filled in prior to cloning). In each case, insertion of a binding element converted the polylinker from binding-negative to binding-positive /data not shown). Both Dist and Dist ${ }^{*}$ give positive results, indicating that the more downstream consensus is functional for binding; the functionality of the more upstream palindrome remains to be tested (but see below).

\section{Receptor binding regions contain functional EcREs}

To establish that these binding regions contain EcREs, we inserted each into a similar location $\sim 240$ bp upstream of the transcriptional start. Our test vector was 
very similar to construct 16 in which the only Eip28/29. derived sequence consists of a short promoter region $(-188$ to +11$)$ driving the cat reporter. In the test vector $\mathrm{X}-188-\mathrm{cc}-\mathrm{cat}$, a long polylinker has been added directly upstream of the Eip segment. As shown in Figure 5B, the vector is ecdysone nonresponsive, giving the usual $20-30 \%$ reduction in cat expression following hormone treatment.

That the hsp27-derived element functions well, giving inductions of $\sim 65$-fold, is confirmation in a new test environment of the observations of Riddihough and Pelham (1987). In addition, each of the receptor-binding sites derived from Eip28/29 functions as an EcRE, though their potencies vary: Element Dist ${ }^{*}$ is most effective (inductions of $\sim 20$-fold), Prox and Dist appear to be intermediate in effect, and Upstream gives marginal, although reproducible, induction. In the case of Prox, two insertion sites differing by $13 \mathrm{bp}$ were tried (Prox- $A$, Prox-B in Fig. 5B|, and a small but probably genuine position effect was observed. Although the activity of Dist has been measured only once, two separate experiments measured the relative effects of Dist ${ }^{*}$ and Dist; Dist $t^{*}$ is evidently 2.4- to 3 -fold more active, suggesting that the more proximal palindrome present in Dist ${ }^{\star}$ contributes to its potency as an EcRE.

To identify the critical nucleotides with greater certainty, the plasmids $11 \mathrm{~N} h s p$ and $15 \mathrm{~N} h s p$ were constructed by synthesizing oligonucleotides whose sequences match the $h s p 27$ 23-mer internally but diverge at the ends. As shown in Figure 5B, $15 \mathrm{~N}$ hsp was fully active as an EcRE; $11 \mathrm{~N} h s p$ was nonfunctional in these assays, and it also failed to bind ecdysone receptor (data not shown). These observations confirm our conclusion that the critical sequence for both receptor-binding and EcRE activity is the palindromic region indicated in Figure 5A.

The data in Figure 5B demonstrate a more remarkable aspect of the hormone response: Ecdysone inducibility arises as a result of both derepression and activation. In every construct containing an EcRE, basal expression is depressed about fourfold relative to the vector. Hormone both alleviates this repression and activates expression to a level substantially above that of the unmodified vector. It is interesting that Upstream, which is a poor EcRE, appears to be equivalent to Dist ${ }^{\star}$ as an inhibitory element. Both aspects of EcRE activity, depression of basal expression and induction by ecdysone, have also been observed in constructs using a heterologous promoter (K. Lee and P. Cherbas, in prep.).

\section{Discussion}

Our principal goal in this work has been to recover EcREs and so begin the process of understanding their functions. We have succeeded in identifying three new examples of short regions bearing functional EcREs, and the properties of these regions along with those of the hsp27 region, studied originally by Riddihough and Pelham (1986), are immediately suggestive.

Sequence comparisons (Fig. 5A) and functional tests
(Fig. 5B) are consistent with the idea that functional EcREs are composed of half-sites of the hexamer $5^{\prime}$ TGA(AC)CY-3', with the most effective elements organized as imperfect palindromes having a single undefined base at the center of symmetry. As described previously, a single restriction cut within this palindrome can obliterate high-affinity receptor binding. Similarly, it is significant that Dist contains two imperfect but palindromic copies of this consensus and that both appear to contribute to its function. The 23-bp region from hsp27 is complex, containing two partially overlapping palindromes. Riddihough and Pelham (1987) focused their attention on a perfect interrupted palindrome composed of the terminal hexamers. Those hexamers bear little similarity to the vertebrate steroid HREs. The test vectors $15 \mathrm{~N} h s p$ and $11 \mathrm{~N} h s p$ demonstrate clearly that the functional feature of the hsp27 23-mer is the central imperfect palindrome, which is also the conserved feature of EcREs. Our data are consistent with either a hexameric or a heptameric half-site, since we have not yet tested the relevant 13-bp cognates. This palindrome is strikingly similar to one group of vertebrate HREs /see below). Similarly, we believe that our conclusions concerning EcRE structure are more compelling than previous inferences (Pongs 1988; Beato 1989), which implicated other portions of the hsp27 23-mer, solely on the basis of sequence comparisons among promoters thought to be ecdysone responsive.

Taken together with the new observation that the ecdysone receptor is a specific DNA-binding protein that interacts with EcREs (see below), the EcRE consensus suggests strongly that ecdysone receptors are members of the steroid receptor superfamily and that ecdysone acts by mechanisms homologous to those for the other steroids. The vertebrate receptors can be classified according to the sequences of their HREs (Evans 1988; Beato 1989). One group (which includes the glucocorticoid, progestin, androgen, and mineralocorticoid receptors) recognizes the half-site $5^{\prime}$-TGTTCT- $3^{\prime}$. A second group /which includes the estrogen, thyroid hormone, retinoic acid, and vitamin $\mathrm{D}$ receptors/ recognizes the half-site $5^{\prime}$-TGACCT- 3 '. This classification is certainly an oversimplification because differences in spacing and small sequence differences are apparently reflected in significant binding specificity (Glass et al. 1988, 1989; Sucov et al. 1990|. Nonetheless, the classification correlates well with sequence relationships among the receptors, particularly with relationships among their DNA-binding domains (Danielson et al. 1989; Mader et al. 1989; Umesono and Evans 1989). Thus, the EcRE consensus suggests a close relationship between the ecdysone receptor and the estrogen, thyroid, retinoic acid, and vitamin $\mathrm{D}$ receptors. Studies of a cloned ecdysone receptor have confirmed this inference (M. Koelle, W. Talbot, W. Segraves, M. Bender, P. Cherbas, and D. Hogness, in prep.).

Although crude receptor extracts labeled by specific steroid binding have been used previously to localize HREs (see, e.g., Pfahl 1982; Glass et al. 1987), the extension of this procedure by blotting has proved to be a par- 
ticularly simple and effective mapping tool. Still, our use of it has thus far been entirely pragmatic and the assay is relatively undefined. Most importantly, our experiments suggest but do not prove that ecdysone receptors bind directly to EcREs. Assuming such binding, the ecdysone receptor is comparable to the other steroid receptors in the magnitude of its DNA specificity as well, because in our assays the presence of a single EcRE approximately doubled the signal due to a 1 - to $2-\mathrm{kb}$ fragment of nonspecific DNA (cf. Pfahl 1982).

Although our binding survey gave no false-positive results - in the sense that all of the sequences identified by blotting proved to be functional EcREs - we know little about its sensitivity and about the frequency of false negative results. On the one hand, Upstream gave a signal indistinguishable from $h s p 27$ despite large differences in their biological effects; on the other, we know that additional EcREs must exist, for example, within region $B$, and that these were not detected. We have occasionally observed intermediate levels of binding by some small DNA fragments (L. Cherbas, unpubl.), but none of these sites has been mapped. The sequenced region around Eip28/29 contains many copies of the halfsite sequence $5^{\prime}$-TGA $/ \mathrm{AC} / \mathrm{CY}-3^{\prime}$, only a few of which tested positive for binding. One possibility is that the blotting assay selectively detects palindromic sequences, but that in vivo "isolated" half-sites can interact over longer distances. Another is that cooperative interactions involving other proteins stabilize some weak EcRE-receptor interactions in vivo but not in our assays.

In any event, until the purified ecdysone receptors become available, the blotting procedure appears to be a practical way to map EcREs. We suspect that with appropriate modifications, it may prove more generally useful in situations where it is possible to use a ligand to label specifically one component of a crude extract.

Although we have identified EcREs in the context of a more general analysis of the hormonal regulation of Eip28/29, our studies of that gene have not yet progressed to the point where we can unambiguously identify the three known EcREs as the necessary elements. Still, it is clear that the ecdysone sensitivity of Eip28/29 depends largely on the presence of sequences downstream of the transcription unit, a result that appears reasonable in light of the insensitivity of the $g d l^{F}$ promoter to the hormone. Similarly, it is satisfying that two of the EcREs recovered fall within regions required for a full ecdysone response (Prox within region $C$ and Dist within region $D$ ). We have noted previously that the third downstream region $(B)$ must contain a weak EcRE that has not yet been identified. For reasons explained earlier we doubt that the stimulatory element(s) in region $A$ is an EcRE; nor does this region exert any obvious effect on basal transcription. Thus, its stimulatory effect on the Eip28/29 ecdysone response is intriguing, particularly because region $A$ falls largely within an independent and nonresponsive transcription unit $\left(g d l^{F}\right)$ and includes its promoter.

The role of Upstream is puzzling, as our experiments have revealed no effect of that element on the Eip28/29 hormone response. Upstream does not fall within any of the known critical regions $(A-D)$. In constructs containing regions $B-D$, inclusion of Upstream does not increase the response (cf. constructs 5-9), and constructs containing only Upstream give no detectable response (cf. constructs 15 and 16). Yet when the Upstream sequence is moved from its normal location $(-453)$ to -188 , it yields a small but easily detectable response. Were Upstream a substantially weaker binding site than Prox or Dist, its inactivity in situ might plausibly be ascribed to distance. Although we cannot eliminate that possibility, we suspect that affinity differences of the magnitude necessary would have been detectable in the binding assays. Interesting alternative explanations are possible: A sequence between -453 and -188 may "block" the effects of receptor bound at Upstream, or a DNA-binding protein whose specificity overlaps that of receptor may compete for binding at Upstream in vivo. Our interest in this problem is considerably enhanced by the observations (Andres 1990) that (1) Eip28/29 is expressed and regulated by ecdysone in numerous tissues of fly larvae; and (2) downstream sequences are required for its regulation in only a subset of those tissues, while sequences upstream of -188 are required in another subset (including epidermis and fat body). Thus, we consider it plausible that Upstream is a functional EcRE under some circumstances and is involved in the regulation of Eip28/29 in some tissues. If so, the specificity might well hinge on the tissue-specific expression of proteins regulating the function of Upstream.

Finally, we turn to the observation that in the absence of hormone, the presence of an EcRE represses expression. The repression itself and the derepression and superactivation induced by hormone are clear in the data of Figure 5B, and all of the effects become still more pronounced when EcREs are introduced as multimers ( $\mathrm{K}$. Lee and P. Cherbas, in prep.). Whether the downstream EcREs have the same effects in situ is an open question. Certainly, this would provide an economical explanation for the elevated basal expression of construct 12 (Table 1 ; Fig. 1A,B), which lacks both of the strong EcREs Prox and Dist. The alternative explanation, that basal expression in that construct is elevated due to the removal of a distinct inhibitory element downstream of region $B$, will require testing by more detailed mutagenesis.

Inhibitory effects of HREs in the absence of hormone are not unknown, the most notable example being the thyroid HRE, which is comparable to an EcRE in its effects (Damm et al. 1989). Although it is possible that the "unliganded" ecdysone receptor binds to an EcRE and represses, we favor the alternative explanation that inhibition results from competition between DNA-binding proteins with overlapping but distinguishable specificities. According to this explanation, the ecdysone-receptor complex displaces an inhibitory protein bound previously at the EcRE. The fact that Upstream gives comparable inhibitions to far stronger EcREs (Fig. 5B) provides some (albeit inconclusive) support for this hy- 
pothesis. There is precedent in the literature for competitive interactions between steroid receptors and other proteins for binding to HREs (Brüggemeier et al. 1990; Crawford and Chapman 1990). It is intriguing to note that the steroid receptor-like proteins encoded by the Drosophila genes E75 (Segraves and Hogness 1990), CF1 (Shea et al. 1990), tll (Pignoni et al. 1990), svp (Mlodzik et al. 1990), kni (Nauber et al. 1988), knrl (Oro et al. 1988), and egon (Rothe et al. 1989) all have sequences suggesting binding to TGACCT-related half-sites (for a summary, see Pignoni et al. 1990). The CF1 product has been shown experimentally to bind to such a sequence (Shea et al. 1990). Thus, it is not only true that each of these proteins is a candidate for the role of the putative inhibitory factor but that a network of competitive regulatory interactions with important developmental consequences may exist.

\section{Materials and methods}

\section{Nomenclature}

We use ecdysone as a generic name for compounds with the appropriate biological activity, in analogy with the terms estrogen or progestin (Cherbas et al. 1984). This reflects common usage. 20-Hydroxyecdysone is the major naturally occurring ecdysone.

\section{Cell lines}

We selected suitable host cell lines after a preliminary survey of Drosophila lines available to us, checking both induction of Eip28/29 and expression of transfected DNA. The lines S2/M3 [(Schneider 1972) adapted by S. Lindquist to growth in M3 medium (Shields et al 1975), supplemented with $10 \%$ fetal calf serum], G2 (a gift from W. Gehring), GM3 (Mosna and Dolfini 1972), DM1 (Schneider 1972; a gift of C. Benyajati), and E$\mathrm{Adh}^{\mathrm{NI}}$ (a line derived from $A d h$-null flies by H.-P. Bernhard; a gift of W. Gehring) exhibited poor induction; the lines S3 (Schneider 1972; a gift of C. Wyss) and $\mathrm{Kc}-\mathrm{H}$ (clone A3Al1; Cherbas et al. 1986b) were very poorly transfectable. Both $\mathrm{Kcl67/M} 3$ and EH34A3/M3 cells gave satisfactory results.

Kc167/M3 cells (Cherbas et al. 1988) were grown in M3 medium (Shields and Sang 1977; as modified by Lindquist et al. 1982) supplemented with $5 \%$ heat-inactivated fetal calf serum. EH34A3/M3 cells were derived from the shibire clone EH34A3 (Woods and Poodry 1983) by adaptation to growth in M3 medium supplemented with $12.5 \%$ heat-inactivated fetal calf serum.

\section{Steroids}

20-Hydroxyecdysone was purchased from Simes spa (Milan), stored, and administered as described previously (Cherbas et al. 1980|. Iodoponasterone labeled with ${ }^{125}$ I was prepared according to Cherbas et al. (1988).

\section{Plasmids}

Unless otherwise specified, constructions were in pUC8 /Vieira and Messing 1982) with the $5^{\prime}$ end of the insert oriented toward the HindIII site of the polylinker. All Eip28/29 fragments were derived from pCg101-1 (Cherbas et al. 1986b) or from the Charon 4 clone Cs500 (Eickbush 1987). Many constructs con- tained tagged Eip28/29 genes in which a 499-bp Sall fragment from bacteriophage $\lambda$ was inserted at a $S n a B I$ site at position +2070 (using an XhoI linker). The $5^{\prime}$ ends of the constructs shown in Figure 2 are an XhoI site at -4600 (construct 11 ), a HindIII site at -2007 (constructs $1,3,4,10,12$, and 14), a PstI site at -657 (constructs 5, 8, and 15), and BAL-31-generated, HindIII-linkered sites at -188 (constructs 7,9 , and 16) and at about -400 (construct 6 , whose $5^{\prime}$ end has not been sequenced). $3^{\prime}$ Ends of the constructs are a HindIII site at +7100 (constructs 1 and 3), a HpaI site at +5882 (constructs 4-9), an Xhol site at +3756 (constructs 10 and 11 ), or a BgIII site at +2484 (constructs 12 and 14). [Coordinates given represent the terminal Eip28/29 base contained in the construct. We note that an earlier statement (Cherbas et al. 1986b) that the region contained no sites for HpaI was in error.]

In constructs 3 and 14 the introns were deleted by substituting a SacI-PstI fragment from the EIP28 cDNA clone pKc45 (Cherbas et al. 1986b) for the corresponding genomic region; this fragment spans all 4 exons of Eip28/29. Eip28/29-cat fusions contain the first 11 bases of the Eip28/29 transcription unit, joined by a short polylinker to the HindIII (filled-in)BamHI fragment of pSV2-cat (Gorman et al. 1982), which contains the structural gene for CAT and an SV40 polyadenylation region. The $3^{\prime}$ Eip28/29 fragment used in constructs 8 and 9 was a SpeI-HpaI fragment consisting of bases +2204 to +5882 , filled in to convert the SpeI site to a blunt end, and inserted into the SmaI polylinker site downstream of the cat-SV40 fragment. Eip188-Adh consists of bases -188 to +11 of Eip28/29, fused by means of a polylinker to a Drosophila Adh fragment extending from base -23 of the larval transcription unit to $\sim 1$ $\mathrm{kb} 3^{\prime}$ of the polyadenylation site (derived from pSAC; Goldberg $1980)$.

X-188-cc-cat, our vector for testing EcREs, was constructed as follows: pUC18 (Yannisch-Perron et al. 1985) was modified by insertion of an additional synthetic polylinker into the HindIII site. This oligonucleotide, 5'-AGCTATCGATAGGCCTGATATCTCGAGATCTCTAGATACGTACGCGTCGACTCGCGA$3^{\prime}$, was inserted with its $3^{\prime}$ end (of the strand shown) toward the pUC18 polylinker. The resulting plasmid, pUCL1, retains all of the sites of $\mathrm{pUCl} 8$ and has additional sites for ClaI, StuI, EcoRV, XhoI, BglII, XbaI, SnaBI, MluI, SalI, and NruI. The insert of 188 -cc-cat (construct 16) was subcloned as a HindIIIBamHI fragment between the HindIII and BamHI sites of pUCLl. Fragments to be tested for EcRE activity (Fig. 5B) were either isolated from digests or synthesized de novo and inserted into one of the polylinker sites upstream of the insert.

The $h s p 27$ promoter region was derived from the clone J1PR 1 (a gift from D. Osterbur, originally from E. Craig). J1PR1 is a subclone of Jl (Craig and McCarthy 1980). The sequence of the region is given by Riddihough and Pelham (1986).

The published sequence for Eip28/29 (Cherbas et al. 1986b) extends only to +2197 . Since elements important for the ecdysone response are not included in this region, we have extended the sequence to +6436 (submitted to GenBank).

\section{Transient expression assays}

Cells at $3 \times 10^{6} / \mathrm{ml}$ (10 ml/100-mm plate) were transfected by the addition of $1 \mathrm{ml} /$ plate $(20 \mu \mathrm{g}$ DNA/plate) of a DNA-calcium phosphate coprecipitate (Wigler et al. 1979); no carrier DNA was used. In all experiments except our initial screen of cell lines for transfectability, we prepared the precipitate at $\mathrm{pH}$ 7.05 rather than $\mathrm{pH} 7.10$; the lower $\mathrm{pH}$ leads to a much stronger ecdysone response for many constructs (K. Lee, unpubl.). Four plates were transfected with each precipitate.

In experiments where expression was assayed by Northern 
analysis, each construct was introduced as an equimolar mixture with Eip188-Adh, with total introduced DNA remaining $20 \mu \mathrm{g} /$ plate. Cells were washed $12 \mathrm{hr}$ after the addition of the precipitate, 20-hydroxyecdysone was added to two of the four plates (final concentration of $10^{-6} \mathrm{M}$ ) $8 \mathrm{hr}$ later, and cells were harvested after an additional $4 \mathrm{hr}$. The contents of duplicate plates were pooled before preparing RNA.

In experiments where expression was assayed by CAT activity, the cells were washed $16 \mathrm{hr}$ after the addition of the precipitate and ecdysone was added to two plates immediately. Each plate was harvested individually $24 \mathrm{hr}$ later. CAT was assayed as described by Gorman et al. (1982) and modified by Rosenthal (1987), corrected for protein concentration (Bio-Rad protein assayl, and CAT specific activities from duplicate plates were averaged. No internal control for transfection was included in these experiments.

\section{Northern analysis}

RNA was isolated by the method of Chomczynski and Sacchi (1987), separated on $1.5 \%$ formaldehyde-agarose gels (Lehrach et al. 1977), using $10 \mu \mathrm{g}$ of RNA per lane, and blotted onto nitrocellulose. Except where otherwise indicated, duplicate Northern blots were probed with ${ }^{32}$ P-labeled RNA probes for the 499-bp SalI fragment of bacteriophage $\lambda$ and for Drosophila $A d h$ to correct for loading errors and RNA degradation, using probes prepared from BlueScribe-based plasmids. Hybridized blots were exposed to Kodak XAR-5 film, without an intensifying screen, and the resulting autoradiograms were quantified by scanning densitometry. Controls for linearity were included in each set of scans.

\section{Kc cell extracts}

Kc167 cells were expanded in spinner flasks and harvested by centrifugation. They were washed once in phosphate-buffered saline $0.13 \mathrm{M} \mathrm{NaCl}, 0.007 \mathrm{M} \mathrm{Na}_{2} \mathrm{HPO}_{4}, 0.003 \mathrm{M} \mathrm{NaH}_{2} \mathrm{PO}_{4}$ at $\mathrm{pH}$ 6.7). In some cases, the cell pellets were frozen in liquid nitrogen and stored at $-80^{\circ} \mathrm{C}$ for several weeks before use; in other cases, they were used fresh. Whole-cell extracts were prepared according to Manley et al. (1983), fast-frozen in small aliquots, and stored at $-80^{\circ} \mathrm{C}$. Aliquots were thawed once and used immediately. Such extracts have a binding capacity of $50-100$ fmoles of steroid per milligram of protein and are stable at $-80^{\circ} \mathrm{C}$ for several months at least.

\section{Blot assays of ecdysone receptor DNA binding}

DNA was cut with restriction enzymes and fractionated by electrophoresis on agarose gels in TAE or on acrylamide gels in TBE (Maniatis et al. 1982). Each lane contained $\sim 1 \mu \mathrm{g}$ DNA/kb of starting material. No ethidium bromide was included in the gels. Following electrophoresis, gels were electroblotted (BioRad TransBlot Cell) onto a GeneScreen Plus filter (New England Nuclear|, using the same buffer system that was used for the initial electrophoresis. For agarose gels, the transfer was at $15 \mathrm{~V}(0.2 \mathrm{~A})$ for $1-2 \mathrm{hr}$, followed by $45 \mathrm{~V}(0.6 \mathrm{~A})$ for $1-2 \mathrm{hr}$. For acrylamide gels, we used $10 \mathrm{~V}(0.04 \mathrm{~A})$, followed by $20 \mathrm{~V}(0.13$ A). Blotting was monitored by including a duplicate gel in which the DNA fragments were either labeled with ${ }^{32} \mathrm{P}$ or stained with ethidium bromide; fragments as small as $80 \mathrm{bp}$ are efficiently blotted by this procedure and are retained on the blot during the ensuing steps. The blot was wrapped in plastic wrap and stored at $-20^{\circ} \mathrm{C}$ until it was probed with the ecdysone receptor. Immediately before treatment with the receptor extract, the blot was shaken for $\sim 1 \mathrm{hr}$ at $4^{\circ} \mathrm{C}$ in milk buffer $(10 \%$ Car- nation nonfat dry milk, $40 \mathrm{~mm} \mathrm{KCl,} 40 \mathrm{~mm}$ HEPES at $\mathrm{pH} 7.0$, $0.1 \mathrm{~mm}$ dithiothreitol).

The ecdysone receptor in a Kc cell extract was labeled with [125I]iodoponasterone (Cherbas et al. 1988) by incubating the undiluted extract with the ligand at $\sim 20 \mu \mathrm{C} / \mathrm{ml}$, at room temperature for $1 \mathrm{hr}$. An aliquot was tested to confirm binding (glass fiber filter technique, as described in Cherbas et al. 1988); typically $\sim 15-20 \%$ of the ligand was bound to receptor. The remainder of the ligand-extract mixture was diluted with 20 volumes of milk buffer and incubated with the blocked DNA blot for $3 \mathrm{hr}$ at $4^{\circ} \mathrm{C}$ with gentle shaking. The volume of the incubation solution was just sufficient to cover the filter, typically 5-10 $\mathrm{ml}$. After incubation with the labeled receptor, the filter was washed at $4^{\circ} \mathrm{C}$ (four changes, each about five times the volume used for incubation, over a period of $2 \mathrm{hr}$ ) in milk buffer, and exposed to Kodak XAR film overnight with an intensifying screen.

\section{Acknowledgments}

This work has been supported by a grant from the National Institutes of Health (GM37813). We are very grateful to L. Hodges and S. Bodayla for technical assistance.

The publication costs of this article were defrayed in part by payment of page charges. This article must therefore be hereby marked "advertisement" in accordance with 18 USC section 1734 solely to indicate this fact.

\section{References}

Andres, A.J. 1990. An analysis of the temporal and spatial patterns of expression of the ecdysone-inducible genes Eip28/29 and Eip40 during development of Drosophila melanogaster. Ph.D. thesis, Indiana University, Bloomington.

Ashburner, M. 1990. Puffs, genes, and hormones revisited. Cell 61: $1-3$.

Ashburner, M., C. Chihara, P. Meltzer, and G. Richards. 1974. Temporal control of puffing activity in polytene chromosomes. Cold Spring Harbor Symp. Quant. Biol. 38: 655662.

Beato, M.J. 1989. Gene regulation by steroid hormones. Cell 56: 335-344.

Bieber, A.J. 1986. Ecdysteroid-inducible polypeptides in Drosophila Kc cells: Kinetics of mRNA induction and aspects of protein structure. Ph.D. thesis, Harvard University, Cambridge.

Brüggemeier, U., L. Rogge, E.-L. Winnacker, and M. Beato. 1990. Nuclear factor I acts as a transcription factor on the MMTV promoter but competes with steroid hormone receptors for DNA binding. EMBO J. 9: 2233-2239.

Carson-Jurica, M.A., W.T. Schrader, and B.W. O'Malley. 1990. Steroid receptor family: Structure and functions. Endocr. Rev. 11: 201-220.

Cherbas, L., C.D. Yonge, P. Cherbas, and C.M. Williams. 1980. The morphological response of $\mathrm{Kc}-\mathrm{H}$ cells to ecdysteroids: Hormonal specificity. Wilhelm Roux's Arch. Dev. Biol. 189: $1-15$.

Cherbas, L., J.W. Fristrom, and J.D. O'Connor. 1984. The action of ecdysone in imaginal discs and Kc cells of Drosophila melanogaster. In Biosynthesis, metabolism and mode of action of invertebrate hormones (ed. J. Hoffmann and M. Porchet), pp. 305-322. Springer-Verlag, Berlin.

Cherbas, L., H. Beneš, M. Bourouis, K. Burtis, A. Chao, P. Cherbas, M. Crosby, M. Garfinkel, G. Guild, D. Hogness, J. Jami, C.W. Jones, M. Koehler, J.-A. Lepesant, C. Martin, F. 
Maschat, P. Mathers, E. Meyerowitz, R. Moss, R. Pictet, J. Rebers, G. Richards, J. Roux, R. Schulz, W. Segraves, C. Thummel, and K. Vijayraghavan. 1986a. Structural and functional analysis of some moulting hormone-responsive genes from Drosophila. Insect Biochem. 16: 241-248.

Cherbas, L., R.A. Schulz, M.M.D. Koehler, C. Savakis, and P. Cherbas. 1986b. Structure of the Eip28/29 gene, an ecdysone-inducible gene from Drosophila. J. Mol. Biol. 189: $617-631$.

Cherbas, P., L. Cherbas, S.S. Lee, and K. Nakanishi. 1988. 26- $\left[{ }^{125} \mathrm{I}\right]$-Iodoponasterone is a potent ecdysone and a sensitive radioligand for ecdysone receptors. Proc. Natl. Acad. Sci. 85: 2095-2100.

Chomczynski, P. and N. Sacchi. 1987. Single-step method of RNA isolation by acid guanidinium thiocyanate-phenolchloroform extraction. Anal. Biochem. 162: 156-159.

Clever, U. 1964. Actinomycin and puromycin: Effects on sequential gene activation by ecdysone. Science 146: 794795.

Craig, E.A. and B.J. McCarthy. 1980. Four Drosophila heat shock genes at 67B: Characterization of recombinant plasmids. Nucleic Acids Res. 8: 4441-4457.

Crawford, L. and K. Chapman. 1990. Identification of a high molecular weight steroid response element binding protein. Mol. Endocrinol. 4: 685-692.

Damm, K., C.C. Thompson, and R.M. Evans. 1989. Protein encoded by v-erbA functions as a thyroid-hormone receptor antagonist. Nature 339: 593-597.

Danielson, M., L. Hinck, and G.M. Ringold. 1989. Two amino acids within the knuckle of the first zinc finger specify DNA response element activation by the glucocorticoid receptor. Cell 57: 1131-1138.

Eickbush, D. 1987. Molecular characterization of the 71CE region of Drosophila melanogaster. Ph.D. thesis, Harvard University, Cambridge.

Evans, R.M. 1988. The steroid and thyroid hormone receptor superfamily. Science 240: 889-895.

Glass, C.K., R. Franco, C. Weinberger, V.R. Albert, R.M. Evans, and M.G. Rosenfeld. 1987. A c-erb-A binding site in rat growth hormone gene mediates trans-activation by thyroid hormone. Nature 329: 738-741.

Glass, C.K., M.J. Holloway, O.V. Devary, and M.G. Rosenfeld. 1988. The thyroid hormone receptor binds with opposite transcriptional effects to a common sequence motif in thyroid hormone and estrogen response elements. Cell 54: $313-323$.

Glass, C.K., S.M. Lipkin, O.V. Devary, and M.G. Rosenfeld. 1989. Positive and negative regulation of gene transcription by a retinoic acid-thyroid hormone receptor heterodimer. Cell 59: 697-708.

Goldberg, D.A. 1980. Isolation and partial characterization of the Drosophila alcohol dehydrogenase gene. Proc. Natl. Acad. Sci. 77: 5794-5798.

Gorman, C.M., L.P. Moffatt, and B.H. Howard. 1982. Recombinant genomes which express chloramphenicol acetyltransferase in mammalian cells. Mol. Cell. Biol. 2: 1044-1051.

Lehrach, H., D. Diamond, J.M. Wozney, and H. Boedtker. 1977. RNA molecular weight determinations by gel electrophoresis under denaturing conditions, a critical reexamination. Biochemistry 16: 4743-4751.

Lindquist, S.L., S. Sonada, T. Cox, and K. Slusser. 1982. Instant medium for Drosophila tissue culture cells. Dros. Inf. Serv. 58: 163.

Mader, S., V. Kumar, H. de Verneuil, and P. Chambon. 1989. Three amino acids of the oestrogen receptor are essential to its ability to distinguish an oestrogen from a glucocorticoid- responsive element. Nature 338: 271-274.

Maniatis, T., E.F. Fritsch, and J. Sambrook. 1982. Molecular cloning: A laboratory manual. Cold Spring Harbor Laboratory, Cold Spring Harbor, New York.

Manley, J.L., A. Fire, M. Samuel, and P.A. Sharp. 1983. In vitro transcription: Whole-cell extract. Methods Enzymol. 101: $568-582$.

Maroy, P., R. Dennis, C. Beckers, B.A. Sage, and J.D. O'Connor. 1978. Demonstration of an ecdysteroid receptor in a cultured cell line of Drosophila melanogaster. Proc. Natl. Acad. Sci. 75: 6035-6038.

Mlodzik, M., Y. Hiromi, U. Weber, C.S. Goodman, and G.M. Rubin. 1990. The Drosophila seven-up gene, a member of the steroid receptor gene superfamily, controls photoreceptor cell fates. Cell 60: $211-224$.

Mosna, G. and S. Dolfini. 1972. New continuous cell lines of Drosophila melanogaster. Morphological characteristics and karyotypes. Dros. Inf. Serv. 48: 144-145.

Nauber, U., M.J. Pankratz, A. Kienlin, E. Seifert, U. Klemm, and H. Jäckle. 1988. Abdominal segmentation of the Drosophila embryo requires a hormone receptor-like protein encoded by the gap gene knirps. Nature 336: 489-492.

Oro, A.E., E.S. Ong, J.S. Margolis, J.W. Posakony, M. McKeown, and R.M. Evans. 1988. The Drosophila gene knirps-related is a member of the steroid-receptor gene superfamily. $\mathrm{Na}$ ture 336: 493-496.

Pfahl, M. 1982. Specific binding of the glucocorticoid-receptor complex to the mouse mammary tumor proviral promoter region. Cell 31: 475-482.

Pignoni, F., R.M. Baldarelli, E. Steingrímsson, R.J. Diaz, A. Patapoutian, J.R. Merriam, and J.A. Lengyel. 1990. The Drosophila gene tailless is expressed at the embryonic termini and is a member of the steroid receptor superfamily. Cell 62: $151-163$.

Pongs, O. 1988. Ecdysteroid-regulated gene expression in Drosophila melanogaster. Eur. J. Biochem. 175: 199-204.

Riddihough, G. and H.R.B. Pelham. 1986. Activation of the Drosophila hsp 27 promoter by heat shock and by ecdysone involves independent and remote regulatory sequences. EMBO I. 5: 1653-1658.

- 1987. An ecdysone response element in the Drosophila hsp27 promoter. EMBO I. 6: 3729-3934.

Rosenthal, N. 1987. Identification of regulatory elements of cloned genes with functional assays. Methods Enzymol. 152: $704-720$.

Rothe, M., U. Nauber, and H. Jäckle. 1989. Three hormone receptor-like Drosophila genes encode an identical DNAbinding finger. $E M B O$ I. 8: 3087-3094.

Sage, B.A., M.A. Tanis, and J.D. O'Connor. 1982. Characterization of ecdysteroid receptors in cytosol and naive nuclear preparations of Drosophila $\mathrm{K}_{\mathrm{c}}$ cells. I. Biol. Chem. 257: 6373-6379.

Savakis, C., G. Demetri, and P. Cherbas. 1980. Ecdysteroid-inducible polypeptides in a Drosophila cell line. Cell 22: 665674.

Savakis, C., M.M.D. Koehler, and P. Cherbas. 1984. cDNA clones for the ecdysone-inducible polypeptide (EIP) mRNAs of Drosophila Kc cells. EMBO J. 3: 235-243.

Schneider, I. 1972. Cell lines derived from late embryonic stages of Drosophila melanogaster. I. Embryol. Exp. Morphol. 27: 353-365.

Schulz, R.A. and B.A. Butler. 1989. Overlapping genes of Drosophila melanogaster: Organization of the z600-gonadalEip28/29 gene cluster. Genes Dev. 3: 232-242.

Schulz, R.A. and J.L. Miksch. 1989. Dorsal expression of the Drosophila $z 600$ gene during early embryogenesis. Dev. 
Biol. 136: 211-221.

Schulz, R.A., W. Shlomchik, L. Cherbas, and P. Cherbas. 1989. The Drosophila Eip28/29 gene and its upstream neighbors: Structural overlap and diverse expression. Dev. Biol. 131: $515-523$.

Segraves, W.A. and D.S. Hogness. 1990. The E75 ecdysone-inducible gene responsible for the $75 \mathrm{~B}$ early puff in Drosophila encodes two new members of the steroid receptor superfamily. Genes Dev. 4: 204-219.

Segraves, W.A. and G. Richards. 1990. Regulatory and developmental aspects of ecdysone-regulated gene expression. Invert. Reprod. Dev. 18: 67-76.

Shea, M.J., D.L. King, M.J. Conboy, B.D. Mariani, and F.C. Kafatos. 1990. Proteins that bind to Drosophila chorion cis-regulatory elements; A new $\mathrm{C}_{2} \mathrm{H}_{2}$ zinc finger protein and a $\mathrm{C}_{2} \mathrm{C}_{2}$ steroid receptor-like component. Genes Dev. 4: 11281140 .

Shields, G. and J.H. Sang. 1977. Improved medium for culture of Drosophila embryonic cells. Dros. Inf. Serv. 52: 161.

Shields, G., A. Dübendorfer, and J. Sang. 1975. Differentiation in vitro of larval cell types from early embryonic cells of Drosophila melanogaster. I. Embryol. Exp. Morphol. 33: $159-175$.

Sucov, H.M., K.K. Murakami, and R.M. Evans. 1990. Characterization of an autoregulated response element in the mouse retinoic acid receptor type $\beta$ gene. Proc. Natl. Acad. Sci. 87: 5392-5396.

Vieira, J. and J. Messing. 1982. The pUC plasmids, an M13mp7derived system for insertion mutagenesis and sequencing with synthetic universal primers. Gene 19: 259-268.

Umesono, K. and R.M. Evans. 1989. Determinants of target gene specificity for steroid/thyroid hormone receptors. Cell 57: 1139-1146.

Wigler, M., A. Pellicer, S. Silverstein, R. Axel, G. Urlaub, and L. Chasin. 1979. DNA mediated transfer of the adenine-phosphoribosyltransferase locus into mammalian cells. Proc. Nati. Acad. Sci. 76: 1373-1376.

Woods, D.F. and C.A. Poodry. 1983. Cell surface proteins of Drosophila. I. Changes induced by 20 - hydroxyecdysone. Dev. Biol. 96: 23-31.

Yanisch-Perron, C., J. Vieira, and J. Messing. 1985. Improved M13 phage cloning vectors and host strains: Nucleotide sequences of the M13mp19 and pUC19 vectors. Gene 33: $103-119$.

Yund, M.A., D.S. King, and J.W. Fristrom. 1978. Ecdysteroid receptors in imaginal discs of Drosophila melanogaster. Proc. Natl. Acad. Sci. 75: 6039-6043. 


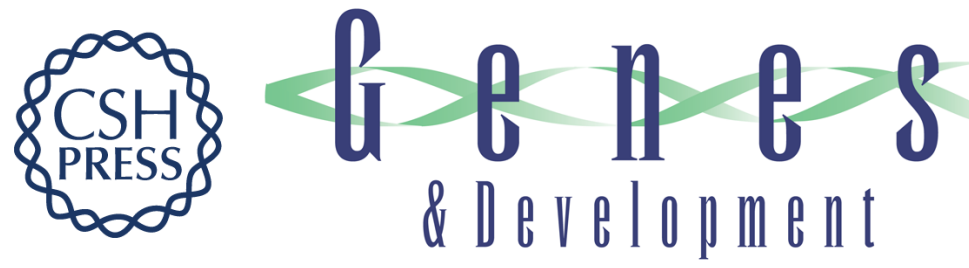

\section{Identification of ecdysone response elements by analysis of the Drosophila Eip28/29 gene.}

$\mathrm{L}$ Cherbas, $\mathrm{K}$ Lee and $\mathrm{P}$ Cherbas

Genes Dev. 1991, 5:

Access the most recent version at doi:10.1101/gad.5.1.120

References This article cites 56 articles, 13 of which can be accessed free at:

http://genesdev.cshlp.org/content/5/1/120.full.html\#ref-list-1

License

Email Alerting

Service

Receive free email alerts when new articles cite this article - sign up in the box at the top right corner of the article or click here.

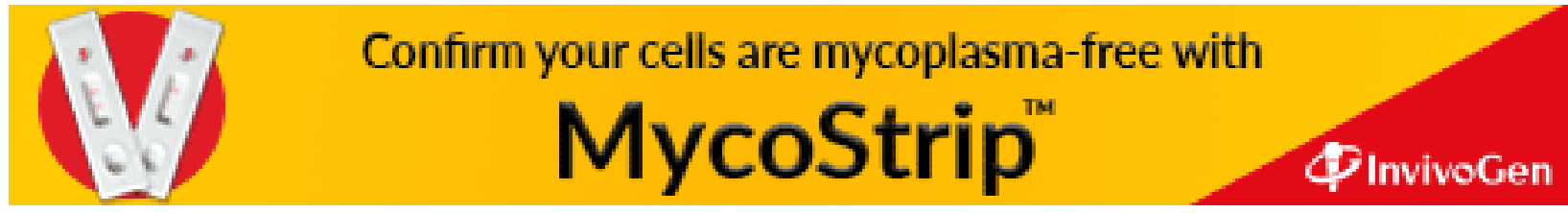

